\title{
A permanent magnetic film atom chip for Bose-Einstein condensation
}

\author{
B.V. Hall, S. Whitlock, F. Scharnberg $\dagger$, P. Hannaford and \\ A. Sidorov \\ ARC Centre of Excellence for Quantum-Atom Optics and Centre for Atom Optics \\ and Ultrafast Spectroscopy, Swinburne University of Technology, Hawthorn, Victoria \\ 3122, Australia \\ E-mail: brhall@groupwise.swin.edu.au
}

\begin{abstract}
.
We present a hybrid atom chip which combines a permanent magnetic film with a micromachined current-carrying structure used to realize a Bose-Einstein condensate. A novel TbGdFeCo material with large perpendicular magnetization has been tailored to allow small scale, stable magnetic potentials for ultracold atoms. We are able to produce ${ }^{87} \mathrm{Rb}$ Bose-Einstein condensates in a magnetic trap based on either the permanent magnetic film or the current-carrying structure. Using the condensate as a magnetic field probe we perform cold atom magnetometry to profile both the field magnitude and gradient as a function of distance from the magnetic film surface. Finally we discuss future directions for our permanent magnetic film atom chip.

PACS number: 39.90.+d,03.75.Be,39.25.+k,07.55.-w
\end{abstract}

\section{Introduction}

A recent technological advance in the area of quantum degenerate gases has been the development of the 'atom chip'. These devices exploit tightly confining, magnetic potentials, created by low power current-carrying wires to simplify the production of Bose-Einstein condensates [1, 2]. In addition, they provide the freedom to realize intricate magnetic potentials with features of size comparable to the atomic de Broglie wavelength. Atom chips have been used to realize atomic waveguides and transport devices for Bose-Einstein condensates [3, 4. These tools allow controllable manipulation of ultracold neutral atoms, with potential applications in quantum information processing [5, 6] and atom interferometry [7, 8].

For current-carrying wire-based atom chips, technical limitations are imposed by current noise and spatial fluctuations in the current density leading to increased heating rates and fragmentation of cold clouds [9, 10]. In addition, near-field thermal noise in conductors is responsible for a fundamental atom loss mechanism [11]. Atom chips $\dagger$ Present address: Institut für Quantenoptik, Universität Hannover, 30167 Hannover, Germany 
incorporating permanent magnetic materials are expected to overcome many of these difficulties. These materials offer the possibility of ultra-stable magnetic potentials due to their intrinsically low magnetic field noise. Moreover permanent magnetic films are thin and relatively high in resistance compared to current-carrying wires, properties which strongly suppress thermal magnetic field noise [12]. Permanent magnetic materials with in-plane magnetization have recently been used to demonstrate trapping of cold atoms [13] and to produce BEC on a magnetic videotape [14, 15]. Here we employ a novel magnetic material with perpendicular anisotropy, developed specifically for applications with ultracold atoms. Perpendicularly magnetized materials allow arbitrary 2D patterns to be written in the plane of the film and provide magnetic field configurations analogous to those produced by planar microfabricated wires [16, 17].

In this paper we report the realization of a permanent magnetic film/machined conductor atom chip which has been used to produce a ${ }^{87} \mathrm{Rb}$ Bose-Einstein condensate (BEC). In section 2 we present a simple model for a thin film of perpendicularly magnetized material which results in straightforward equations for the magnetic field near the edge of the film. We then describe the principle of trapping ultracold atoms in the potential formed by the permanent magnetic film (the film trap). TbGdFeCo materials are then introduced in section 3 with a description of the deposition process. We measured the bulk properties of the magnetic film using both a superconducting quantum interference device (SQUID) and a magnetic force microscope (MFM). Section 4 describes the construction of the ultra high vacuum (UHV) compatible atom chip. This includes a current-carrying structure to provide time dependent control of surface based potentials and is used to form a conductor-based magnetic trap (the wire trap).

The apparatus and experimental procedures used for making a BEC independently with the film trap or the wire trap are described in section 5. In section 6 we apply the BEC as a novel ultracold atom magnetometer by measuring the spatial decay of the magnetic field from the film. High precision trap frequency measurements in conjunction with radio frequency output coupling also allow the direct determination of the associated magnetic field gradient. In conclusion we speculate on future directions for our permanent magnetic film atom chip.

\section{Simple model of a permanent magnetic film}

Consider a semi-infinite rectangular magnet with magnetization $M$ and thickness $h$ (Figure 1). The magnet lies in the $x y$ plane with one edge aligned along the $y$ axis. The magnet is uniformly magnetized in the $+z$ direction and the distance from the film is large with respect to the film thickness $(z \gg h)$. The magnetic field and the field gradient directly above the edge can be written as

$$
B_{\text {film }}=\frac{\mu_{0}}{2 \pi} \frac{h M}{z} \text { and } B_{f i l m}^{\prime}=-\frac{\mu_{0}}{2 \pi} \frac{h M}{z^{2}} .
$$

These expressions are analogous to those derived using Biot-Savart's law for the magnetic field above an infinitely long and thin current-carrying wire. The similarity 


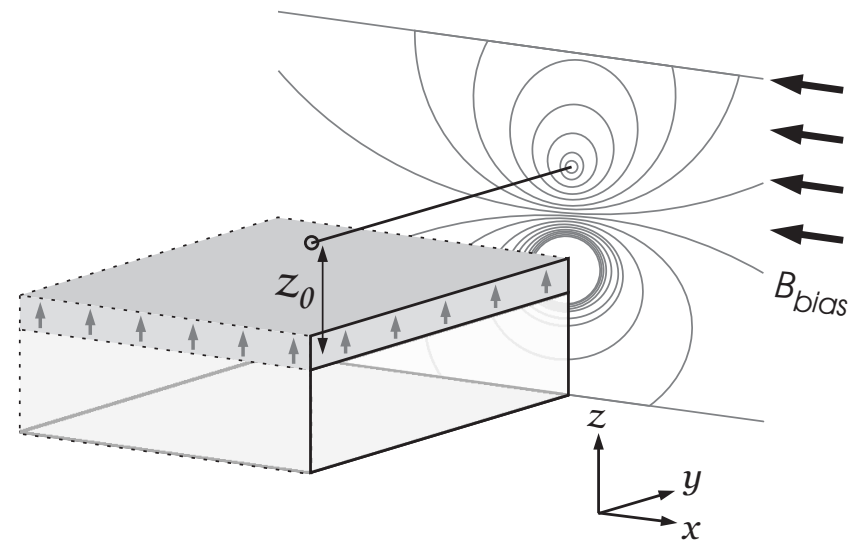

Figure 1. A simple model describes the magnetic field of a semi-infinite, perpendicularly magnetized thin film in combination with a uniform bias magnetic field.

between a permanent magnetic film and a current-carrying wire can be explained using a simple model. An unmagnetized film is comprised of many small magnetic domains of random orientation. The magnetic field produced by each domain is equivalent to that from an imaginary surface current flowing along the domain borders, perpendicular to the magnetization vector [18]. For a uniformly magnetized film with perpendicular anisotropy all domains are aligned in the same direction (out of plane) and within the bulk the magnetic fields of neighbouring domains cancel. A net effective current exists about the perimeter of the film with a magnitude given by the product of the magnetization and the film thickness $\left(I_{e f f}=h M\right)$.

The application of a uniform bias field $\left(B_{\text {bias }}\right)$ in the $-x$ direction produces a radially symmetric two-dimensional quadrupole magnetic field above the film edge at the height $z_{0}$, where the magnitudes of $B_{\text {bias }}$ and $B_{\text {film }}$ are equal. To realize a threedimensional (3D) magnetic trap for weak-field seeking atoms a nonuniform axial field $B_{y}$ is provided by two parallel currents located beneath and perpendicular to the waveguide. Additionally, $B_{y}$ suppresses spin-flip losses by preventing the total magnetic field at the trap bottom from going to zero. This results in a 3D harmonic film trap at a distance $z_{0}$ from the surface with radial frequency given by

$$
2 \pi f_{\text {radial }}=\frac{\mu_{0}}{2 \pi} \frac{h M}{z_{0}^{2}} \sqrt{\frac{\mu_{B} g_{F} m_{F}}{m B_{y}}},
$$

where $\mu_{B}$ is the Bohr magneton, $g_{F}$ is the Landé factor, $m_{F}$ is the magnetic quantum number and $m$ is the atomic mass. The ability to produce high quality, thick magnetic films with large magnetization is necessary to produce tightly confining magnetic traps for ultracold atoms. 


\section{3. $\mathrm{Tb}_{6} \mathrm{Gd}_{10} \mathrm{Fe}_{80} \mathrm{Co}_{4}$ magneto-optical films and their properties}

The desire for large capacity information storage devices has encouraged an extensive investment toward developing novel magnetic compositions. These are primarily optimized to achieve small scale, recordable patterning of magnetic media. While it is possible to benefit from this experience, applications with cold atoms have several additional yet very specific requirements. Firstly, a high Curie temperature $\left(T_{C}\right)$ will prevent demagnetization during the bake-out procedure, a necessary step in achieving UHV conditions. Secondly, a high coercivity $\left(H_{C}\right)$ will prevent the loss of magnetization when applying large external magnetic fields. Finally, the remanent magnetization $\left(M_{R}\right)$ and the saturation magnetization $\left(M_{S}\right)$ should be large and nearly equivalent, an indication of good magnetic homogeneity. These conditions are satisfied by $\mathrm{Tb}_{6} \mathrm{Gd}_{10} \mathrm{Fe}_{80} \mathrm{Co}_{4}$ magneto-optical films which have a high Curie temperature $\left(T_{C} \approx 300^{\circ} \mathrm{C}\right)$, perpendicular anisotropy and a square hysteresis loop.

TbGdFeCo films were produced using a thin film deposition system (Kurt J Lesker CMS-18) equipped with magnetron sputtering and electron beam evaporation sources [19]. A composite target with a nominal atomic composition of $\mathrm{Tb}_{6} \mathrm{Gd}_{10} \mathrm{Fe}_{80} \mathrm{Co}_{4}$ and a high purity chromium target are used in the production of the magnetic films. A systematic study of the influence of process parameters over the properties of the film indicated that deterioration of the magnetic anisotropy occurs for film thickness above $250 \mathrm{~nm}$. In order to maintain good magnetic properties and increase the magnetic field strength near the surface we have implemented a multilayer deposition which produces high quality TbGdFeCo magnetic films with a total thickness approaching $1 \mu \mathrm{m}$. A glass slide substrate was cleaned in an ultrasonic bath using a nitric acid solution then carefully rinsed before being mounted in the deposition chamber. The base pressure was less than $5 \times 10^{-8}$ Torr prior to introducing the argon buffer gas ( $\sim 4$ mTorr). The substrate was then heated to $100{ }^{\circ} \mathrm{C}$ and a bonding layer of chromium $(120 \mathrm{~nm})$ was sputtered on the surface. This was followed by the deposition of six bi-layers of TbGdFeCo $(150 \mathrm{~nm})$ and $\mathrm{Cr}(120 \mathrm{~nm})$ films.

The magnetic properties of the multilayer $\mathrm{TbGdFeCo} / \mathrm{Cr}$ film were characterized by a SQUID magnetometer (Figure 21). The hysteresis loop indicates a remanent magnetization of $0.28 \mathrm{~T}$ for a total magnet thickness of $900 \mathrm{~nm}(h M=0.20 \pm 0.01 \mathrm{~A})$. Complete magnetization of the film can be achieved by applying a field of $\sim 0.8 \mathrm{~T}$, while the film magnetization is robust in the presence of external fields below $\sim 0.1 \mathrm{~T}$. The surface features of the films have also been examined by a high-resolution atomic force microscope operating in magnetic force mode (Figure 31). An unmagnetized sample shows micron-sized features consistent with domain stripes, while an example of a uniformly magnetized sample exhibits excellent magnetic homogeneity. 


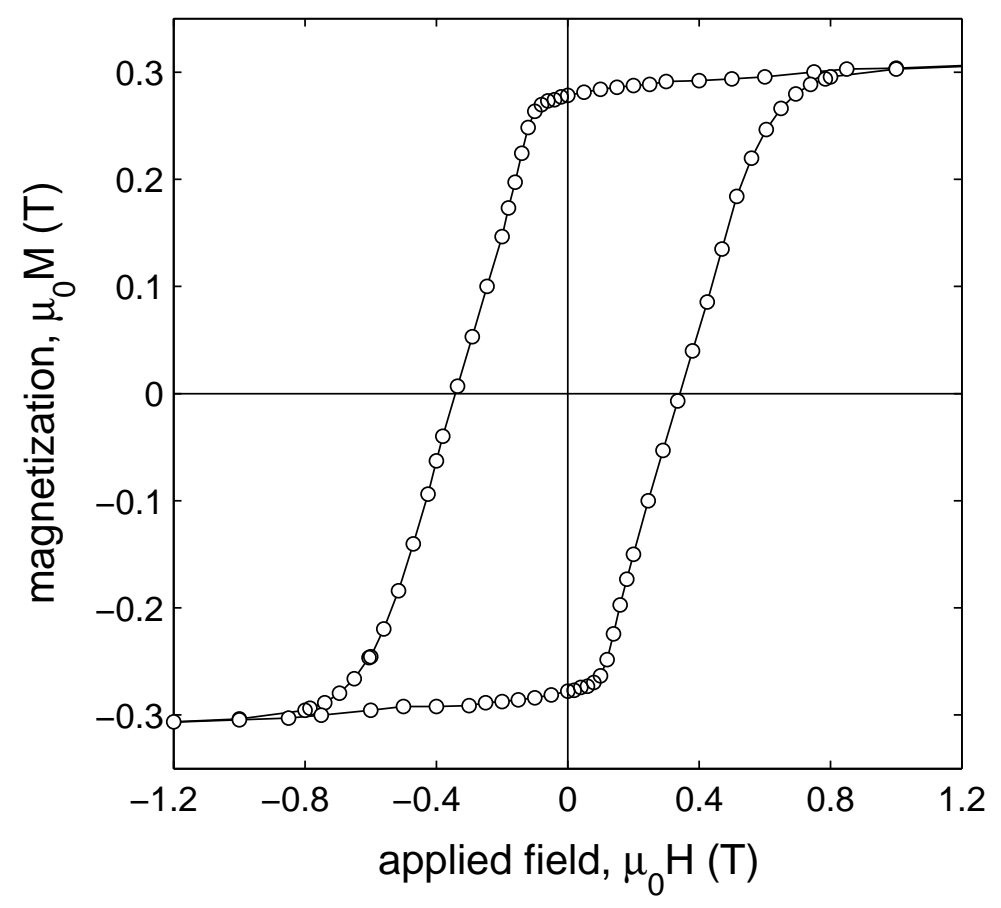

Figure 2. A hysteresis loop derived from SQUID magnetometry of a multilayer $\mathrm{Tb}_{6} \mathrm{Gd}_{10} \mathrm{Fe}_{80} \mathrm{Co}_{4}$ magnetic film. The film magnetization is $\mu_{0} M_{S} \sim \mu_{0} M_{R}=0.28 \mathrm{~T}$ and the coercivity is $\mu_{0} H_{C}=0.32 \mathrm{~T}$.
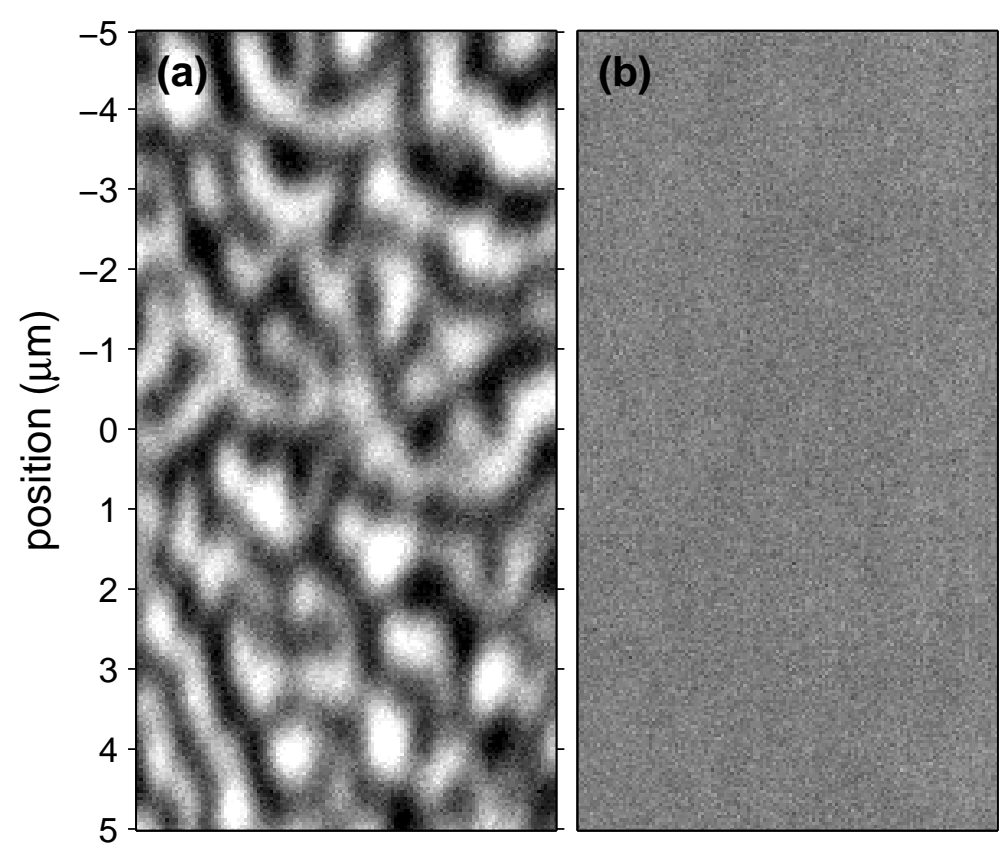

Figure 3. Magnetic Force Microscope (MFM) image of a $\mathrm{Tb}_{6} \mathrm{Gd}_{10} \mathrm{Fe}_{80} \mathrm{Co}_{4}$ magnetic film surface. (a) Unmagnetized sample shows domain structure with micron sized features. (b) Uniformly magnetized sample is free of any visible magnetic structure. 


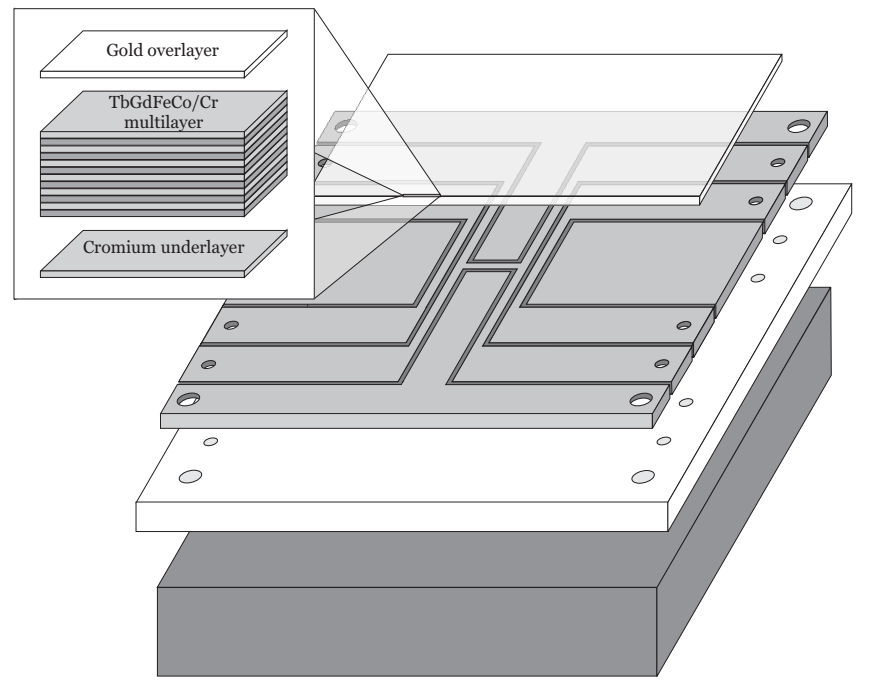

Figure 4. Schematic view of the hybrid atom chip. Inset: TbGdFeCo/Cr multilayer film and $\mathrm{Au}$ overlayer. From the top down, glass slide coated with magnetic film, machined silver foil $\mathrm{H}$-wire and end wires, Shapal-M base-plate and $\mathrm{Cu}$ heat sink. Missing from the schematic is the second glass slide and two rubidium dispensers

\section{Atom chip design}

This device represents the first atom chip based on a perpendicularly magnetized permanent magnetic film for trapping ultracold atoms. It has been designed for the production and manipulation of a BEC near the surface of the magnetic material. Although these films are well suited for making tight and stable trapping potentials up to a few $100 \mu \mathrm{m}$ from the surface, the small volume of the film trap is not suitable for efficient loading directly from a magneto-optical trap (MOT). To circumvent this difficulty a current-carrying wire structure located beneath the magnetic film provides an additional trapping field. The combination of both the magnetic film and the wire structure represents the hybrid atom chip design shown schematically in Figure 4 ,

The top layer of the atom chip consists of two adjacent $300 \mu \mathrm{m}$ thick glass slides which are sturdy enough to prevent warping. The long edges of the glass slides were polished with aluminium oxide grit prior to deposition to remove visible chips. A multilayer $\mathrm{TbGdFeCo} / \mathrm{Cr}$ film was deposited on one slide using the procedure outlined in Section 3. Both slides were then coated with a gold overlayer $(170 \mathrm{~nm})$ and together form a large reflective surface $\left(40 \times 46 \mathrm{~mm}^{2}\right)$. This allows the collection of a large number of atoms into a mirror MOT within a single-chamber UHV system. The glass slide coated with the $\mathrm{TbGdFeCo} / \mathrm{Cr}$ multilayer film was then magnetized in a uniform field of $1 \mathrm{~T}$ pending assembly.

The second layer of the hybrid chip is a wire structure which was produced using the micro-machined silver foil technique developed by Vale et al [20. A $500 \mu \mathrm{m}$ thick silver foil (99.99 \% purity) was fixed with epoxy (Epotek H77) to a $2 \mathrm{~mm}$ thick Shapal-M 
machineable ceramic base-plate. A computer controlled Quick Circuit 5000 PCB mill was used to cut $500 \mu \mathrm{m}$ wide insulating grooves in the foil. Each wire has a width of $1 \mathrm{~mm}$ which is broadened to $6 \mathrm{~mm}$ far from the trapping region to facilitate good electrical connections. After cutting, the insulating channels were filled with additional epoxy to increase the structural integrity and thermal conductivity. The wire structure including electrical connections has a total resistance of $4.6 \mathrm{~m} \Omega$. A continuous current of $30 \mathrm{~A}$ can be applied with an associated temperature rise of less than $40{ }^{\circ} \mathrm{C}$ and negligible increase in vacuum pressure.

In conventional atom chips $\mathrm{U}$ or Z-shape wires are used for creating quadrupole and Ioffe-Pritchard $(I P)$ magnetic field geometries to realize mirror MOTs and magnetic microtraps 21. In the present atom chip, high currents are used to form a tight trap relatively far from the wire, thereby avoiding unwanted collisions with the surface of the slide. Consequently, the use of broad conductors prohibit the use of separate U

and Z-shape wires. This is circumvented with a planar H-shape structure, designed to allow both U and Z-shape current paths with good spatial overlap of the associated traps. Axial confinement for the film trap is provided by additional parallel conductors separated by $9.5 \mathrm{~mm}$ and located either side of the H-shape structure. The top surface of the machined silver foil was later polished flat to support the glass slides.

During assembly, the polished edge of the TbGdFeCo film coated slide is aligned to the middle of the H-shape structure and set with epoxy. The second gold-coated slide is epoxied adjacent to the magnetic film slide to complete the reflective chip surface. Two rubidium dispensers are mounted on two ceramic blocks (Macor) which are recessed below the chip surface. The two glass slides, machined silver foil and ceramic base-plate are then fixed to a copper heat sink. The completed chip is clamped to a $19 \mathrm{~mm}$ diameter solid copper feedthrough (Ceramaseal, 800 A rating) and mounted in the vacuum chamber. Electrical connections are made using $1.6 \mathrm{~mm}$ diameter bare copper wire and $\mathrm{BeCu}$ barrel connectors in conjunction with a 12 pin power feedthrough (Ceramaseal, 55 A rating). A cold cathode gauge indicated a pressure below $1 \times 10^{-11}$ Torr after baking at $140{ }^{\circ} \mathrm{C}$ for 4 days, highlighting the UHV compatibility of all materials.

\section{Bose-Einstein condensation on a permanent magnetic film}

The reflective surface of the atom chip is used to form a mirror MOT and accommodates $30 \mathrm{~mm}$ diameter laser beams provided by a high-power diode laser (Toptica DLX110) locked to the $\mathrm{D}_{2}(F=2 \rightarrow 3)$ cooling transition of ${ }^{87} \mathrm{Rb}$. The trapping light is detuned $18 \mathrm{MHz}$ below resonance and has an intensity of $4 \mathrm{~mW} / \mathrm{cm}^{2}$ in each beam. A repumping laser locked to the $\mathrm{D}_{2}(F=1 \rightarrow 2)$ transition is combined with the trapping light with an intensity of $0.5 \mathrm{~mW} / \mathrm{cm}^{2}$ per beam. Two water-cooled coils mounted outside the vacuum chamber provide a quadrupole magnetic field with gradient $0.1 \mathrm{~T} / \mathrm{m}$ centered $4.6 \mathrm{~mm}$ below the chip surface. To load the mirror MOT a current of $6.5 \mathrm{~A}$ is pulsed for $9.5 \mathrm{~s}$ through one resistively heated $\mathrm{Rb}$ dispenser, allowing the collection of $2 \times 10^{8}$ atoms. The atoms are held for a further $15 \mathrm{~s}$ while the UHV pressure recovers, ready for transfer to the chip-based potentials. 


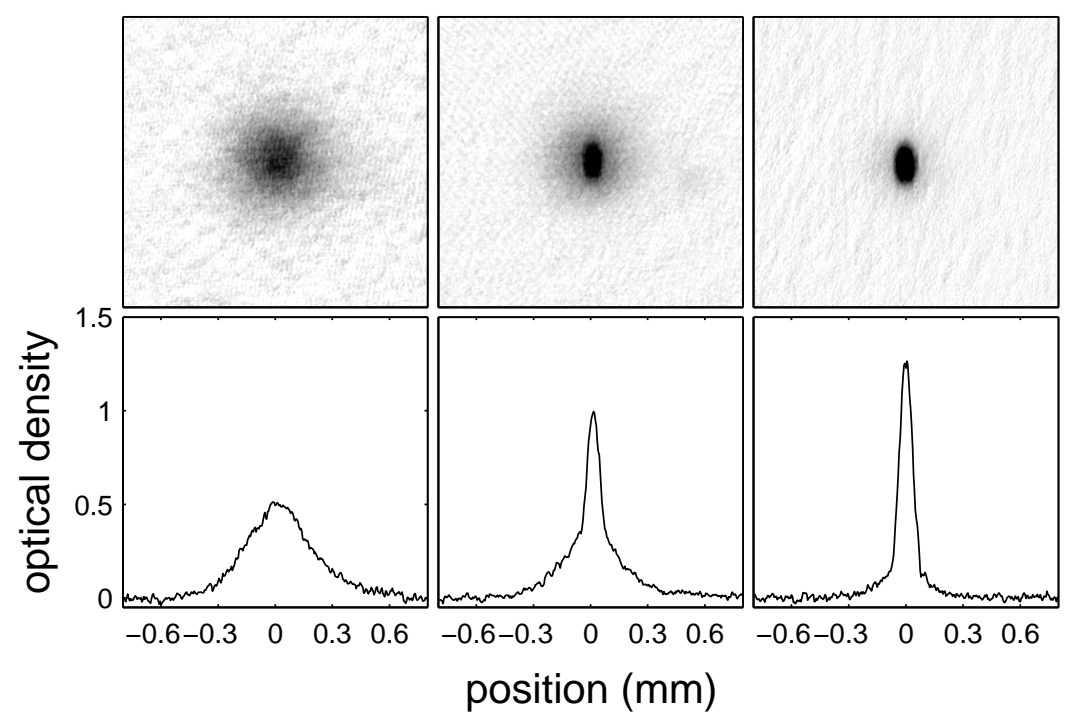

Figure 5. Typical absorption images and optical density profiles of a ballistically expanded atom cloud. Each image is a single realization of the experiment where evaporation is performed in the permanent magnetic film potential. After truncating the evaporation ramp, atoms are held for $150 \mathrm{~ms}$ and ballistically expanded for $30 \mathrm{~ms}$ before imaging. (a) $\mathrm{RF}_{\text {final }}=804 \mathrm{kHz}$ - thermal cloud, (b) $\mathrm{RF}_{\text {final }}=788 \mathrm{kHz}$ - partially condensed cloud, (c) $\mathrm{RF}_{\text {final }}=760 \mathrm{kHz}$ - an almost pure condensate.

Transfer begins by simultaneously ramping a current through the U-shape circuit $\left(I_{U}=0 \rightarrow 8 \mathrm{~A}\right.$ ), increasing the uniform field $B_{\text {bias }}$ and turning off the external quadrupole magnetic field over $50 \mathrm{~ms}$. This moves the atoms without loss, into a U-wire MOT located $1.6 \mathrm{~mm}$ from the surface and increases the radial gradient to $0.4 \mathrm{~T} / \mathrm{m}$. While this compression increases the spatial overlap with the $I P$ potential, it also heats the cloud. To counteract this, the radial gradient is reduced rapidly to $0.11 \mathrm{~T} / \mathrm{m}$ with the trap light off to minimize any force on the atoms. Polarization gradient cooling is applied for $2 \mathrm{~ms}$ with $56 \mathrm{MHz}$ red-detuned trap light to reduce the temperature from $140 \mu \mathrm{K}$ to $40 \mu \mathrm{K}$. Both the MOT light and $I_{U}$ are then turned off leaving the cold atoms in a uniform magnetic field.

Next a $200 \mu$ s optical pumping pulse is applied to maximize the number of atoms in the $\left|F=2, m_{F}=+2\right\rangle$ weak-field seeking state ready for magnetic trapping. A current $\left(I_{Z}\right)$ of $21.5 \mathrm{~A}$ is switched on through the Z-shape circuit while $B_{\text {bias }}$ is increased to $1.3 \mathrm{mT}$ to form an $I P$ wire trap at the same position. A total of $4 \times 10^{7}$ atoms are held with a background-limited lifetime greater than $60 \mathrm{~s}$. Adiabatic compression of this trap is performed by ramping $I_{Z}$ up to $31 \mathrm{~A}$ and $B_{\text {bias }}$ up to $4.0 \mathrm{mT}$ over $100 \mathrm{~ms}$. Further compression results in loss of atoms to the surface. The compressed magnetic trap is $560 \mu \mathrm{m}$ from the film surface where the radial and axial trap frequencies are $2 \pi \times 530 \mathrm{~Hz}$ and $2 \pi \times 18 \mathrm{~Hz}$ respectively. The elastic collision rate in this trap $\left(\gamma_{e l} \approx 50 \mathrm{~s}^{-1}\right)$ is high enough to begin evaporative cooling. 
Forced evaporative cooling to the BEC transition begins in the wire trap and is then transferred to the film trap during a single logarithmic radio frequency (RF) ramp. The first $8.85 \mathrm{~s}$ of this ramp is a preliminary cooling stage in the wire trap down to a temperature of $\sim 5 \mu \mathrm{K}$. As the cloud is cooled the trap is compressed further to improve the evaporation efficiency by lowering $\mathrm{I}_{Z}$ to $25 \mathrm{~A}$, moving the trap to $350 \mu \mathrm{m}$ from the surface and increasing the radial trap frequency to $\sim 2 \pi \times 660 \mathrm{~Hz}$. The RF amplitude is then reduced to zero for $150 \mathrm{~ms}$ while the atoms are transferred closer to the chip surface and finally to the film trap. In this trap $I_{Z}$ is zero and axial confinement on the magnetic film edge is provided by the two end wires, each with a current of $6 \mathrm{~A}$. The trap bottom is tuned using an additional magnetic field parallel to the film edge to minimize any discontinuity in the $\mathrm{RF}$ evaporation trajectory. The radial and axial trap frequencies are $2 \pi \times 700 \mathrm{~Hz}$ and $2 \pi \times 8 \mathrm{~Hz}$ respectively. The $\mathrm{RF}$ amplitude is then increased again and evaporation continues for $1 \mathrm{~s}$ to the BEC phase transition.

Before imaging, the magnetic film trap is adiabatically moved $0.17 \mathrm{~mm}$ from the surface to avoid excessive field gradients from the film. The cloud is then released by switching off $B_{\text {bias }}$ and the atoms fall under gravity with minor acceleration from the permanent field gradient. Resonant optical absorption is used to image the atoms with a $100 \mu \mathrm{s} \sigma^{+}$light pulse parallel to the gold surface and tuned to the $\mathrm{D}_{2}(F=2 \rightarrow 3)$ transition. A CCD camera records the absorption image of the cloud using an achromatic doublet telescope with a resolution of $5 \mu \mathrm{m} /$ pixel. Using the above procedure a new condensate of $1 \times 10^{5}$ atoms is created every $50 \mathrm{~s}$. Figure 5 shows absorption images and optical density profiles after $30 \mathrm{~ms}$ of ballistic expansion. The forced RF evaporation is truncated at $804 \mathrm{kHz}, 788 \mathrm{kHz}$ and $760 \mathrm{kHz}$ revealing a thermal cloud, partially condensed cloud and nearly pure condensate, respectively.

It is also possible to form a condensate trapped solely by the wire trap. Here a single, uninterrupted, $10 \mathrm{~s}$ RF ramp results in a BEC with atom number comparable to that realized in the film trap. This provides a unique possibility for studying the properties of a BEC in both permanent magnetic and current-carrying trapping environments. In addition, the formation of a BEC independent of the top layer will allow new magnetic structures or materials to be replaced with ease. The wire trap can also be used to transport a BEC to regions on the chip where the magnetic field topology may be different from those near the substrate edge.

\section{Magnetic field characterization}

The magnetic properties of the TbGdFeCo film were measured prior to mounting on the atom chip using a combination of SQUID and magnetic force microscopy. In-situ

techniques using cold atoms have been employed to characterize the magnetic field produced by the film inside the vacuum chamber. This allows a direct comparison with the simple model described earlier. A magnetically trapped cloud of cold atoms or a BEC behaves as an ultra-sensitive probe to the local magnetic field. A measure of the trap position as a function of $B_{b i a s}$ determines $B_{\text {film }}(z)$, while an independent measure 
of the trap frequency is used to determine $B_{f i l m}^{\prime}(z)$.

Once the BEC is confined by the film trap it is possible to profile the magnetic field dependence near the surface. The potential minimum is located at the point where the uniform magnetic field is equal in magnitude to and cancels the field from the film $\left(B_{\text {bias }}=-B_{\text {film }}\right)$. The uniform magnetic field can be increased (decreased) to move the trap minimum closer to (further from) the film surface. The BEC follows the potential minimum and the measurement of cloud position with respect to the film surface determines $B_{\text {film }}(z)$. The strength of $B_{\text {bias }}$ is calibrated within the vacuum chamber using untrapped atoms (far from the film) and a short RF pulse resonant with the Zeeman splitting. The pixel size in the imaging plane is calibrated against the gravitational acceleration of freely falling atoms and agrees with the calibration given by imaging a reference rule external to the apparatus. Unfortunately though, the glass substrate coated with magnetic material has recessed approximately $50 \mu \mathrm{m}$ behind the second blank glass slide as a consequence of unevenly cured epoxy. The exact position of the film surface (in relation to the image) is therefore unknown and presents an uncertainty in $B_{\text {film }}(z)$. For this reason a second technique has been applied to provide more information about the magnetic field from the film.

Harmonic oscillations with small amplitude and frequencies up to $10 \mathrm{kHz}$ can be measured accurately over many periods with a BEC due to low damping rates and small spatial extent. In this case, trap frequencies are measured by exciting radial center of mass motion within the film trap and have been measured to better than $1 \mathrm{~Hz}$ ( $\sim 0.1 \%$ accuracy). These excitations were observed by rapidly increasing the uniform magnetic field by approximately $5 \%$ before returning to the original position within 2 to $5 \mathrm{~ms}$. The cloud position was measured after $10 \mathrm{~ms}$ of free expansion and data has been taken over five periods of oscillation. In addition, the trap bottom was measured using RF outcoupling with an accuracy better than $10 \mathrm{mG}(\sim 1 \%)$. The measurement of trap frequency in combination with the trap bottom $\left(B_{y}\right)$ unambiguously determines the local magnetic field gradient (see Equation 2). This combined with the trap position measurements have been used to provide the magnetic field and the magnetic field gradient as a function of height above the surface (Figure 6). This data is consistent with a prediction based on the simple model where the film thickness-magnetization product is given by the prior SQUID measurement ( $h M=0.20 \mathrm{~A})$.

\section{Discussion and conclusion}

We have demonstrated a hybrid atom chip that exploits perpendicularly magnetized film or current-carrying wires for the production of a BEC. We have developed a

multilayer magnetic film structure $(\mathrm{TbGdFeCo} / \mathrm{Cr})$ that provides large magnetization and thickness, important for realizing tight and flexible magnetic microtraps. We have used the BEC as a sensitive probe to directly measure the local magnetic field and gradient associated with the magnetic film. These measurements justify the use of the simple model for perpendicularly magnetized magnetic microstructures. 

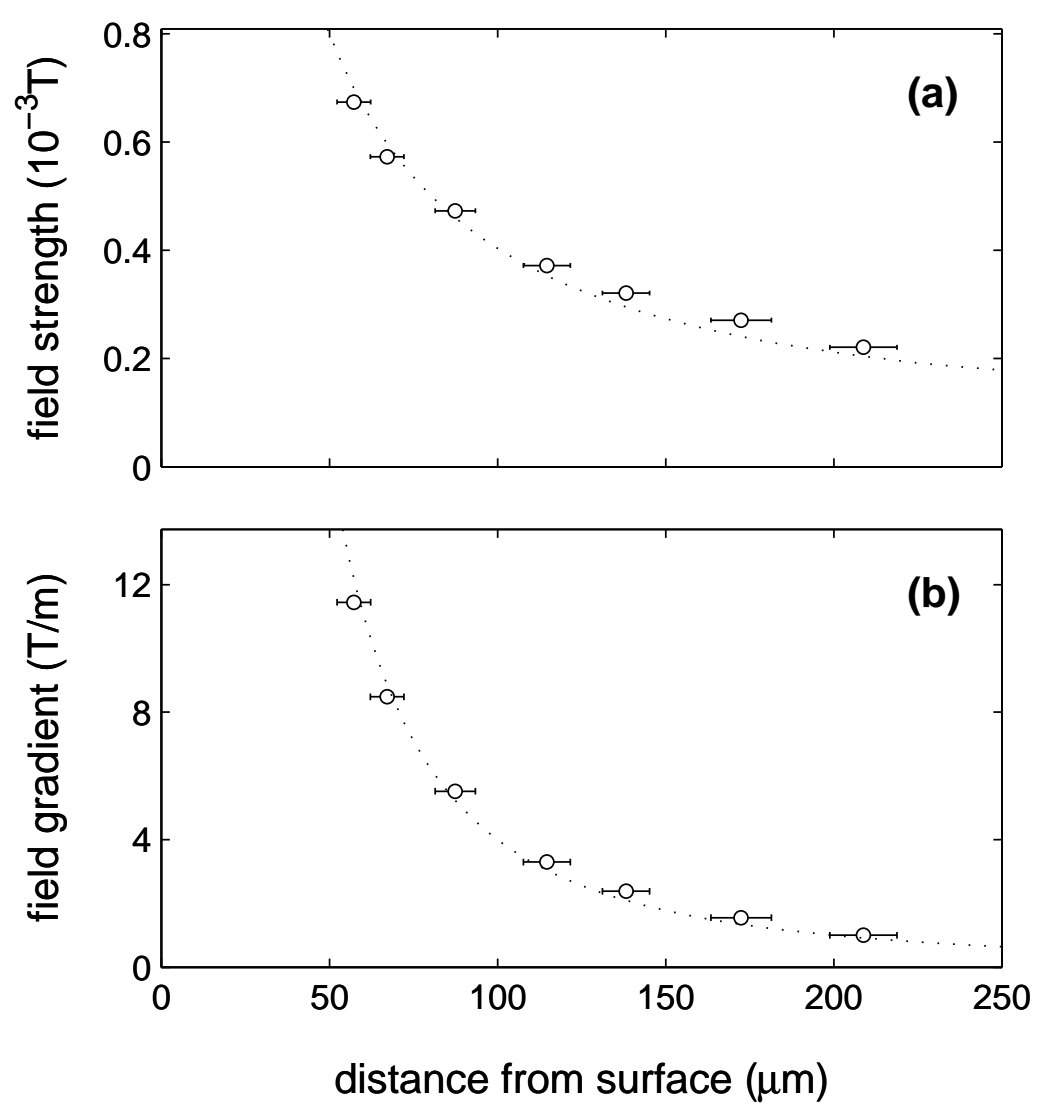

Figure 6. Measurements of the magnetic field strength (a) and field gradient (b) as a function of distance from the surface. The data (open circles) agrees well with predictions (dotted line) of the simple model (see Equation 1). Experimental errors are mostly determined by image resolution and a small uncertainty in the pixel size calibration.

At present we are extending the technique of cold atom magnetometry to the measurement of the spatial dependence of the magnetic field along the film edge. Spatially dependent magnetic field variations have been observed above microfabricated wire-based atom chips and have been attributed to spatial deviations along the wire edge [10, 22]. Similar phenomena observed in permanent magnetic structures may be caused by substrate roughness, deposition irregularity or ultimately domain reversal. Future studies are aimed at the interaction between a BEC and magnetic thin films. A comparison of the decoherence rates of condensates confined in either the film or wire-based microtraps may reveal intriguing possibilities for coherent manipulation of cold atoms in microstructured permanent magnetic potentials.

\section{Acknowledgments}

We would like to thank J. Wang and D. Gough for carrying out the magnetic film deposition. This project is supported by the ARC Centre of Excellence for Quantum- 
Atom Optics and a Swinburne University Strategic Initiative fund.

\section{References}

[1] Hänsel W, Hommelhoff P, Hänsch T W and J Reichel J 2001 Nature 413 498-501

[2] Ott H, Fortágh J, Schlotterbeck G, Grossmann A and Zimmermann C 2001 Phys. Rev. Lett. 23 230401

[3] Leanhardt A, Chikkatur A, Kielpinski D, Shin Y, Gustavson T, Ketterle W and Pritchard D 2002 Phys. Rev. Lett. 89040401

[4] Müller D, Anderson D Z, Grow R J, Schwindt P D D and Cornell E A 1999 Phys. Rev. Lett. 83 5194

[5] Calarco T, Hinds E A, Jaksch D, Schmiedmayer J, Cirac J I and Zoller P 1999 Phys. Rev. A 61 022304

[6] Wang Y-J, Anderson D Z, Bright V M, Cornell E A, Diot Q, Kishimoto T, Prentiss M, Saravanan R A, Segal S R and Wu S 2005 Phys. Rev. Lett. 94090405

[7] Shin Y, Sanner C, Jo G-B, Pasquini T A, Saba M, Ketterle W and Pritchard D E 2005 arXiv:cond-mat/0506464

[8] Schumm T, Hofferberth S, Andersson L M, Wildermuth S, Groth S, Bar-Joseph I, Schmiedmayer $\mathrm{J}$ and Krüger P 2005 arXiv:quant-ph/0507047

[9] Fortágh J, Ott H, Kraft S, Günther A and Zimmermann C 2002 Phys. Rev. A 66041604

[10] Estève J, Aussibal C, Schumm T, Figl C, Mailly D, Bouchoule I, Westbrook C I and Aspect A 2004 Phys. Rev. A $\mathbf{7 0} 043629$

[11] Jones M P A, Vale C J, Sahagun D, Hall B V and Hinds E A 2003 Phys. Rev. Lett. 91080401

[12] Scheel S, Rekdal P K, Knight P L and Hinds E A 2005 arXiv:quant-ph/0501149

[13] Barba I, Gerritsma R, Xing Y T, Goedkoop J B and Spreeuw R J C 2005 Eur. Phys. J. D 00055-3

[14] Sinclair C D J, Curtis E A, Llorente Garcia I, Retter J A, Hall B V, Eriksson S, Sauer B E and Hinds E A 2005 Preprint arXiv:cond-mat/0503619

[15] Sinclair C D J, Curtis E A, Llorente Garcia I, Retter J A, Hall B V, Eriksson S, Sauer B E and Hinds E A 2005 Preprint arXiv:physics/0502073

[16] Eriksson S, Ramirez-Martinez F, Curtis E A, Sauer B E, Nutter P W, Hill E W and Hinds E A 2004 Appl.Phys. B $\mathbf{7 9} 811$

[17] Jaakkola A, Shevchenkon A, Lindfors K, Hautakorpi M, Ilyashenko E, Johansen T H and Kaivola M 2005 Eur. Phys. J. D 00176-7

[18] Jackson J D 1999 Classical Electrodynamics 3rd Edition (New York: Wiley) chap 5

[19] Wang J Y, Whitlock S, Scharnberg F, Gough D S, Sidorov A I, McLean R J and Hannaford P 2005 submitted J. Phys. D

[20] Vale C J, Upcroft B, Davis M J, Heckenberg N R and Rubinsztein Dunlop H 2004 J. Phys. B: At. Mol. Opt. Phys. 3729592967

[21] Reichel J, Hänsel W and Hänsch T D 1999 Phys. Rev. Lett. 833398

[22] Wang D, Lukin M and Demler E 2004 Phys. Rev. Lett. 92076802 